\title{
Data-driven profiling of traffic flow with varying road conditions
}

\author{
O K Golovnin ${ }^{1}$ \\ ${ }^{1}$ Samara National Research University, Moskovskoe Shosse 34A, Samara, Russia, 443086 \\ e-mail: golovnin@ssau.ru
}

\begin{abstract}
The article describes the road, institutional and weather conditions that affect the traffic flow. I proposed a method for traffic flow profiling using a data-driven approach. The method operates with macroscopic traffic flow characteristics and detailed data of road conditions. The article presents the results of traffic flow speed and intensity profiling taking into account weather conditions. The study used road traffic and conditions data for the city of Aarhus, Denmark. The results showed that the method is effective for traffic flow forecasting due to varying road conditions.
\end{abstract}

\section{Introduction}

Modern technical traffic control systems integrate the means of collecting, storing and analyzing data coming from various technical devices (vehicle sensors, radar detectors, video cameras, road weather stations) into a monitoring system. The monitoring system continuously takes into account a number of parameters: the speed and presence of vehicles, air and roadway temperature, speed and direction of the wind. The data collected in the automatic mode are used for adaptive control of the traffic flow using various traffic flow models, which allow the profiling of changes in the traffic flow characteristics from certain influences [2].

There are three classes of traffic flow models. The macroscopic model describes the traffic flow in terms of averaged characteristics [3]: density, intensity, average speed. With this approach, the traffic flow, moving along the road network, is modeled as a fluid movement [4]. Microscopic models describe the traffic flow as detailed as possible [5]: the movement of each vehicle is calculated individually. The microscopic model allows to achieve high adequacy of the traffic flow description compared to the macroscopic model, but it will require large computational resources [6]. An intermediate place is occupied by mesoscopic models [7], in which the traffic flow is described as consisting of an individual vehicle, but the characteristics of their movement are averaged. An important property of mesoscopic traffic flow models is based on both micro and macro indicators [8].

To study the behavior of traffic flow in various situations, the complexity of the traffic flow model is introduced by injecting additional parameters [9]. The described traffic flow models focus on the study of traffic flow in a separate straight section of the street-road network, while the cause of traffic jams, according to [10], are "bottlenecks" formed not only by the street-road network structure but also various road conditions. Therefore, it is important to use the traffic flow model that adequately describes the traffic flow behavior during the passage of "bottlenecks". 
For example, to study the behavior of the traffic flow when a density of saturation and congestion is reached, a parameter describing the passage time of the street-road network equal to the length of the vehicle moving with free movement speed is introduced [11]. For solving the tasks of ensuring traffic safety, an equivalent distance can act as an additional parameter, which decreases with increasing speed (at the same density), indicating the situation becomes more complicated. In [12], a traffic flow model was proposed, based on the Tanaka model, but taking into account the speed limit in the city to meet the safe traffic requirements. In [13], a prediction model based on the composition of machine learning and time series was proposed. In [14], an approach was proposed to take into account the influence of several static bottlenecks on the traffic flow.

With the growing number and composition of data used by modern innovative traffic management tools, traffic flow models should be modified [15]. Thus, traffic flow models begin to depend on data and be controlled by them (data-driven) [16]. The purpose of the work is to systematically analyze data obtained from various sources (traffic flow sensors, weather stations, video cameras, open media, etc.) to profile the impact of road, organizational and weather factors on the traffic flow characteristics.

\section{Profiling of the road conditions impact on traffic flow}

\subsection{Road conditions}

On a large scale, road conditions directly or indirectly affecting traffic flow are shown in figure 1. All other weather, organizational and road conditions, one way or another, are reduced to aggregated conditions.

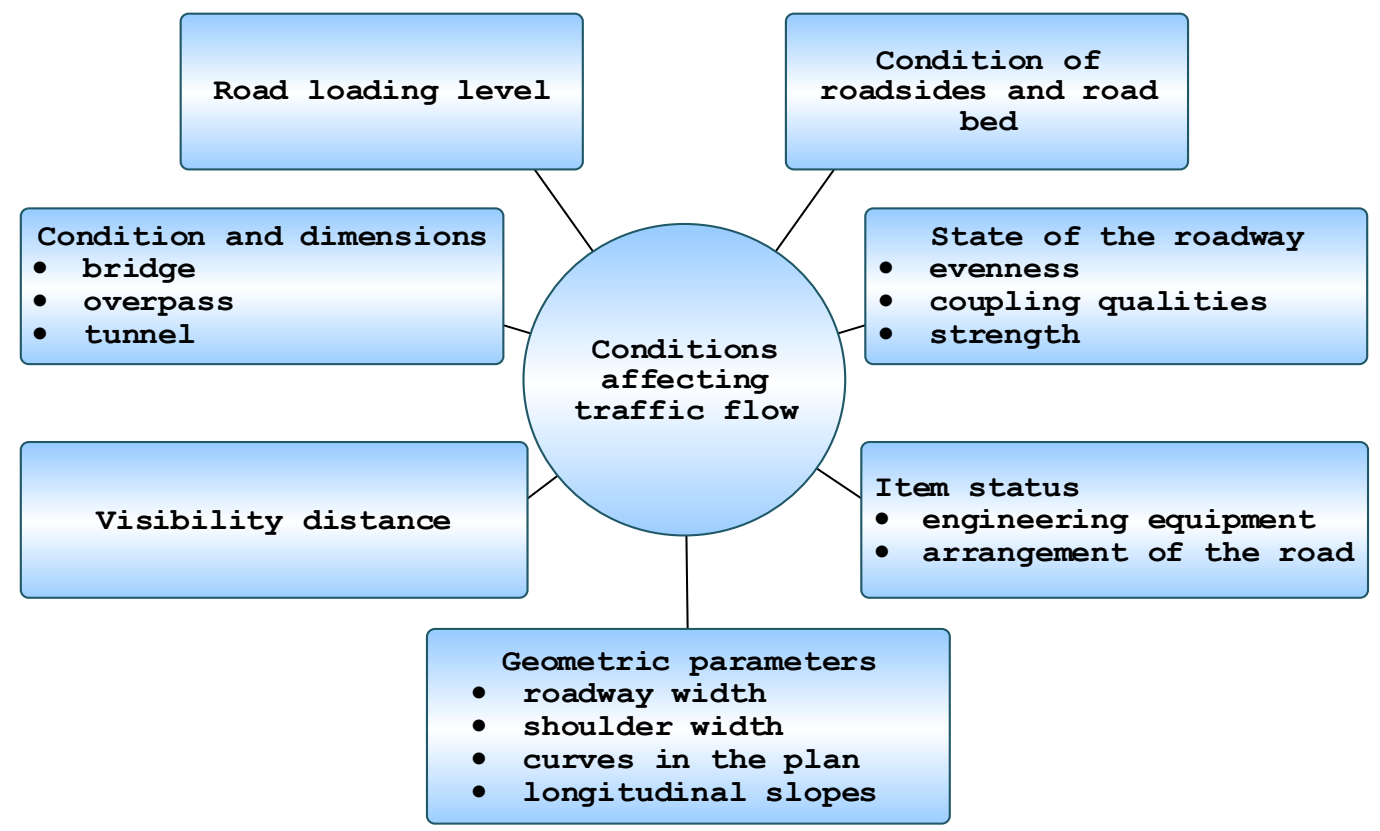

Figure 1. Road conditions affecting traffic flow.

The variety of road conditions and their influence on the traffic flow characteristics is taken into account using a function:

$$
Y=\alpha_{0} \prod_{i=1}^{n} x_{i}^{\lambda_{i}}
$$

where $Y$ is the parameter characterizing the traffic flow (average speed, intensity, density); $x_{i}-$ the street-road network characteristic or road conditions affecting the final indicator $Y ; \lambda_{i}$ - degree of influence of the $x_{i}$ characteristic on $Y ; \alpha_{0}$ - reduction parameter. 


\subsection{Traffic flow}

In the task of profiling the impact of road conditions on the traffic flow, it is not necessary to isolate a separate vehicle from the stream, since aggregated conditions affect the traffic flow generally, which causes the use of a macroscopic model.

In a macroscopic model, the traffic flow parameters are interconnected by the basic equation displayed by the fundamental diagram, the dependence function of the three main macroparameters: average speed $v(t, x)$, intensity $I(t, x)$, density $k(t, x)$ :

$$
\begin{gathered}
v(t, x)=\frac{I(t, x)}{k(t, x)}, \\
I(t, x)=\frac{\partial Q}{\partial t}, \\
k(t, x)=\frac{\partial Q}{\partial x}, \\
I(t, x)=f(k(t, x)),
\end{gathered}
$$

where $Q$ is the number of vehicles approaching the street-road network section.

The used fundamental diagram is shown in figure $2[10,17]$.

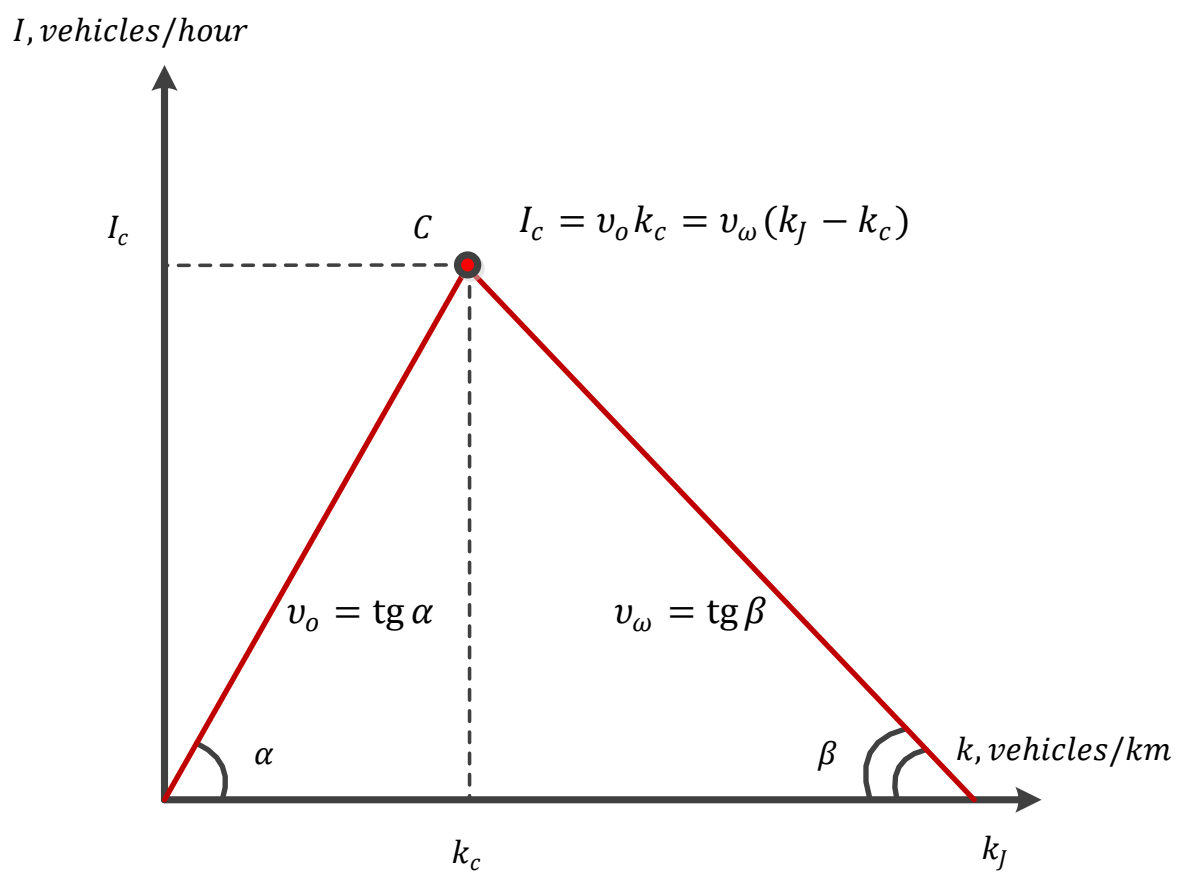

Figure 2. Fundamental traffic flow diagram.

\subsection{Method of profiling the impact of road conditions on traffic flow}

The method of profiling the impact of road conditions on traffic flow consists of the following steps (figure 3).

Step 1. Formation of a street-road network model.

The model is formed from a variety of areas $\Theta=\left\{\theta_{i}^{X}\right\}$ of different types with a single set of attributes (spans $\Theta^{L}$, intersections $\Theta^{S}$, tunnels $\Theta^{U}$, overpasses $\Theta^{O}$, railroad crossings $\Theta^{R}$, pedestrian crossings $\Theta^{P}$ ), multiple arcs $\tilde{E}=\left\{\tilde{e}_{i}\right\}$ and multiple nodes $\tilde{V}=\left\{\tilde{v}_{i}\right\}$. 


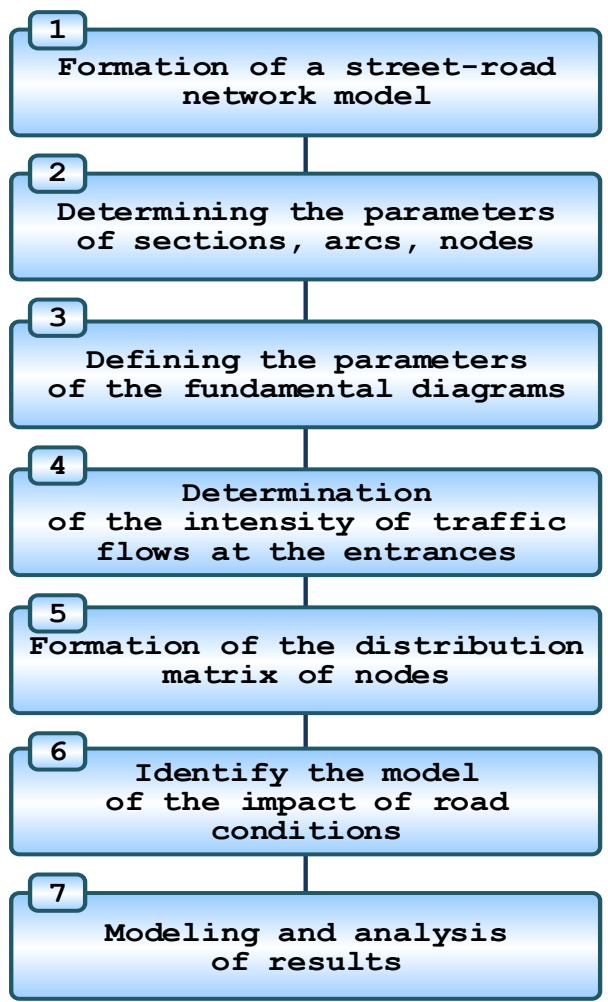

Figure 3. Steps of profiling the impact of road conditions on traffic flow.

Step 2. Determining the parameters of sections, arcs, nodes.

The parameters of the graph street-road network, shown in figure 1, are identified.

Step 3. Defining the parameters of the fundamental diagrams.

Determination of flow macromodel (2)-(5) for identification of fundamental diagrams (triads $I_{C}{ }^{\tilde{e}_{i}}, v_{0}^{\tilde{e}_{i}}, v_{w}^{\tilde{e}_{i}}$ or $I_{C}{ }^{\tilde{e}_{i}}, k_{C} \tilde{e}_{i}, k_{J}^{\tilde{e}_{i}}$ ) for arcs $\tilde{e}_{i} \in \tilde{E}$ of the street-road network graph. This step is performed using the street-road network and technical means of monitoring, such as sensors and video monitoring systems, databases of field surveys, radar detectors and presence vehicle detectors.

For arcs of the street-road network $\tilde{e}_{i} \in \tilde{E}$, where the data on the traffic flow parameters are missing or unreliable, the main traffic flow parameters are defined as follows. The maximum intensity value $I^{\tilde{e}_{i}}$ on the arc $\tilde{e}_{i}$ among the available measurement (observation) results of intensity $I^{\tilde{e}_{i}} \in \widetilde{I}^{\tilde{e}_{i}}$ is selected as the arc bandwidth $I_{C} \tilde{e}_{i}$.

Free flow speed on an $\operatorname{arc} v_{0}^{\tilde{e}_{i}}$ is equal to the maximum speed of movement in this street-road network section defined by the Road Traffic Regulations or other regulatory documents of the location country.

Critical density $k_{C} \tilde{e}_{i}$ traffic flow on an arc is calculated by:

$$
k_{C}^{\tilde{e}_{i}}=\frac{I_{C}^{\tilde{e}_{i}}}{v_{0}^{\tilde{e}_{i}}} .
$$

Maximum traffic flow density on the $\operatorname{arc} k_{J}^{\tilde{e}_{i}}$ is established by estimating the maximum number of vehicles $Q^{\tilde{e}_{i}}$ that can fit on the arc $\tilde{e}_{i}$ : 


$$
k_{J}^{\tilde{e}_{i}}=\frac{Q^{\tilde{e}_{i}}}{l^{\tilde{e}_{i}}} .
$$

This method of estimating the maximum density $k_{J}^{\tilde{e}_{i}}$ can be applied with a high degree of accuracy in the presence of aerial photographs obtained using remote Earth sensing methods, on which a congestion state is recorded. An example of an arc throughput calculation is shown in Figure 4.

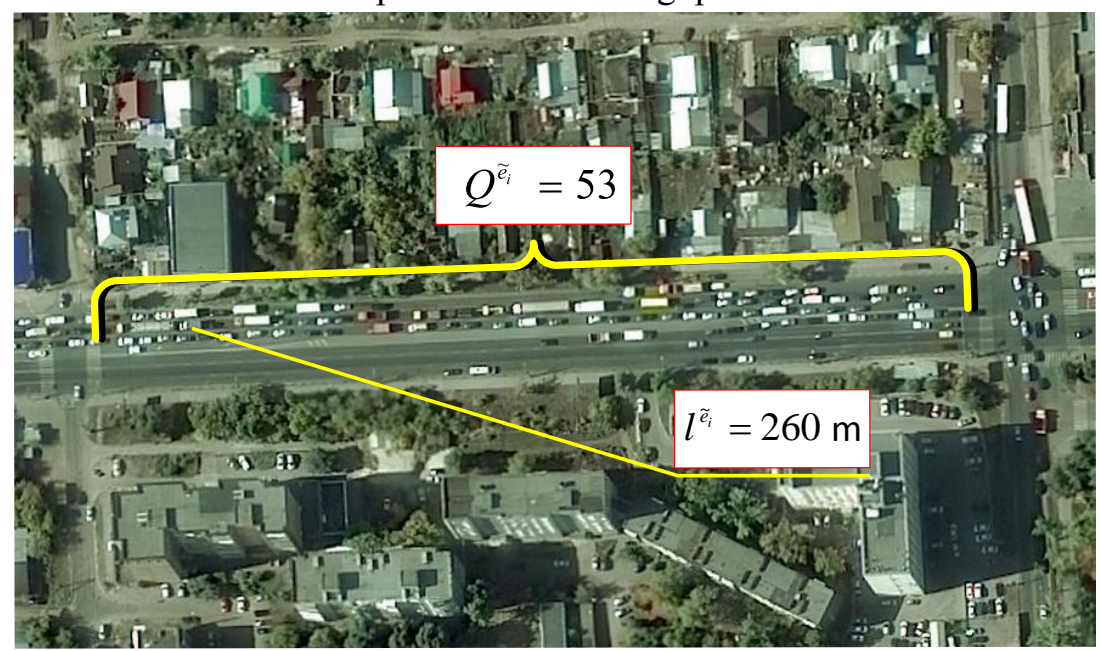

Figure 4. Arc throughput calculation example.

If the intensity, density or average speed sensors are installed on the street-road network, then using the least squares method applied to points with known traffic flow intensity and density in the fundamental diagram, I find the speed of free flow $v_{0}^{\tilde{e}_{i}}$ and the speed of propagation of the traffic jam $v_{w}^{\tilde{e}_{i}}$ along the arc.

If the parameters of the traffic flow in the surveyed area street-road network cannot be established, then I use the parameters of the traffic flow from the neighboring sections street-road network to synthesize the missing data.

Step 4. Determination of the intensity of traffic flows at the entrances.

To determine the intensity of the traffic flow at the entrances $I_{E}^{\tilde{e}_{i}}(t)=I_{R} \tilde{e}_{i}(t)$ to the street-road network, I use an algorithm that generates values according to Poisson's law and takes into account the number of inhabitants and the level of motorization in the transport area.

Step 5. Formation of the distribution matrix of nodes.

Formation of distribution matrices $\mathrm{B}^{\tilde{v}_{i}}$ for all nodes street-road network $\tilde{v}_{i} \in \tilde{V}$ will be performed using an algorithm analyzing the sets of traffic flow intensity values $\tilde{I}^{\tilde{e}_{m}}, \tilde{I}^{\tilde{e}_{n}}$ on $\operatorname{arcs} \tilde{e}_{m}$ entering the node $\tilde{v}_{i}$ and $\operatorname{arcs} \tilde{e}_{n}$ leaving the node $\tilde{v}_{i}$ under study, respectively.

Step 6. Identify the model of the impact of road conditions.

The model of the impact of road conditions is implemented according to (1).

Step 7. Modeling and analysis of results

By changing the parameters of the fundamental diagrams of arcs $I_{C}^{\tilde{e}_{i}}, v_{0}^{\tilde{e}_{i}}, v_{w}^{\tilde{e}_{i}}, I_{C}^{\tilde{e}_{i}}, k_{C}{ }^{\tilde{e}_{i}}, k_{J}^{\tilde{e}_{i}}$ I simulate weather phenomena, incidents, control actions. Changes in the intensity at the arcs of entrances $I_{R}^{\tilde{e}_{i}}(t)$ simulate fluctuations in transport demand arising from the effects of attraction points. By changing the distribution matrix $\mathrm{B}^{\widetilde{v}_{i}}(t)=\left(\beta_{m, n}(t)\right)_{m=1 . n=1}^{M, N}$ in the node $\tilde{v}_{i}$, the traffic flow redirection is modeled, for example, due to the use of information support tools for traffic participants. 


\section{Results}

\subsection{Identification of the traffic flow model}

Due to the simplicity of identification, I use the fundamental diagram proposed by $[10,17]$, which is determined by the maximum road capacity $I_{C}$, the free flow speed $v_{0}$ and the speed of propagation of the traffic jam $v_{w}$ (figure 2).

Basic traffic flow diagrams, built on the well-known classical models for experimental data, I present in figure 5. All models assume a saturation point, where the intensity reaches its maximum value. With a further increase in traffic flow density, the intensity decreases.

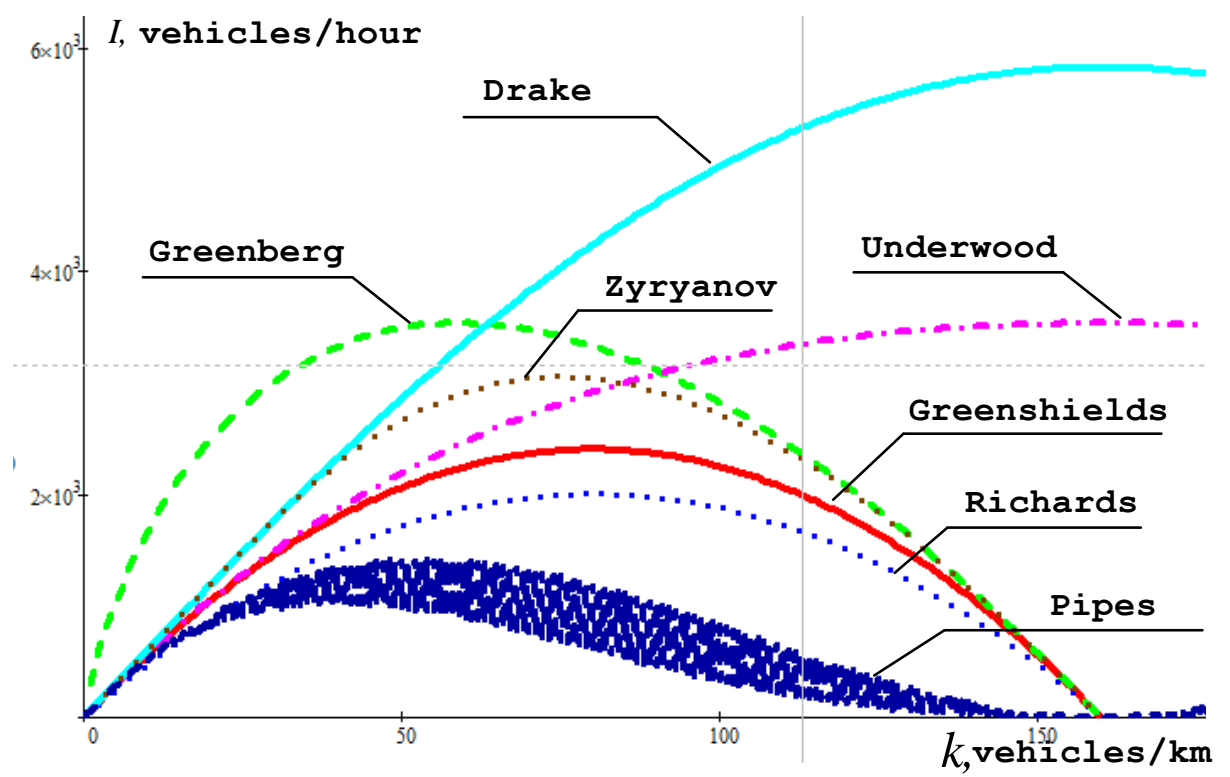

Figure 5. Comparison of the fundamental diagrams of macromodels.

I use the data on average speed and intensity (vehicle number per time) of traffic flow, on the basis of which the density of the traffic flow can be calculated.

\subsection{Software implementation}

Software that implements the proposed method operates in the .NET Framework 4.5 and is written in C\#. The graphical user interface is based on WinForms and integrates into the attribute-driven network-centric intelligent transport system [18]. The interaction with the services of the intelligent transport system was accomplished through the SOAP protocol using WCF technology.

Data on traffic flow and road conditions for analysis are obtained from the CityPulse road database for the city of Aarhus, Denmark [19, 20]. Data are presented in CSV and JSON format. Interval of measurement of traffic flow characteristics -5 minutes. I take environmental measurements every 1 st, 20th and 50th minute.

A traffic flow entry contains data:

- measurement status (presence of errors);

- average time of passage of the site street-road network (s);

- average speed $(\mathrm{km} / \mathrm{h})$;

- vehicle count.

The road conditions record contains weather data:

- dew point $\left({ }^{\circ} \mathrm{C}\right)$;

- humidity $(\%)$

- atmospheric pressure (millibar);

- temperature $\left({ }^{\circ} \mathrm{C}\right)$; 
- $\quad$ direction of the wind $\left({ }^{\circ}\right)$;

- wind speed $(\mathrm{km} / \mathrm{h})$.

Data are converted into developed software using continuous integration and import feature that provides control over data integrity and consistency [21, 22]. Data are accompanied by associated attribute data, which are the semantics of real-world objects. For systems operating on the basis of the platform with an electronic map, the attribute data are basic, the geodata provide a spatial reference. Data are presented in the form of domain objects (Domain Object pattern) or data transfer objects (DTO pattern). To load related data in these objects, I use the Lazy Load design pattern, which works through WCF using SerializationSurrogate, which is attached by metaprogramming methods.

\subsection{Application results}

In the developed software, a series of experiments were conducted with the obtained data set consisting of 25 million records. The results obtained using the proposed method allowed me to establish weather conditions that would help to achieve maximum $\mathrm{I}_{\max }=1538 \mathrm{vehicles} / \mathrm{h}$ and minimum $I_{\min }=12$ vehicles $/ \mathrm{h}$ values of intensity and maximum $\mathrm{V}_{\max }=150.5 \mathrm{~km} / \mathrm{h}$ and minimum $\mathrm{V}_{\min }$ $=11.6 \mathrm{~km} / \mathrm{h}$ values of average speed (table 1 ).

Table 1. Weather conditions for extremes of the traffic flow characteristics.

\begin{tabular}{lcccccc}
\hline & $\begin{array}{c}\text { Dew point } \\
\left({ }^{\circ} \mathrm{C}\right)\end{array}$ & Humidity $(\%)$ & $\begin{array}{c}\text { Pressure } \\
(\text { millibar })\end{array}$ & $\begin{array}{c}\text { Temperature } \\
\left({ }^{\circ} \mathrm{C}\right)\end{array}$ & $\begin{array}{c}\text { Wind } \\
\text { direction }\left({ }^{\circ}\right)\end{array}$ & $\begin{array}{c}\text { Wind speed } \\
(\mathrm{km} / \mathrm{h})\end{array}$ \\
\hline $\boldsymbol{I}_{\max }$ & 1 & 87 & 1003 & 3 & 140 & 7.4 \\
$\boldsymbol{I}_{\min }$ & 17 & 88 & 1012 & 19 & 280 & 11.1 \\
$\boldsymbol{V}_{\max }$ & 11 & 73 & 1013 & 15 & 180 & 5.6 \\
$\boldsymbol{V}_{\min }$ & 9 & 88 & 1013 & 11 & 210 & 5.6
\end{tabular}

The influence results of individual weather conditions on the traffic flow: in table 2 - the effect of the dew point deficit, in table 3 - the atmospheric pressure effect, in table 4 - the wind speed. The smallest (Min), highest (Max) and average (Avg) values describing the influence of weather conditions on the traffic flow are considered. In this case, the maxima and minima of the values of the average speed and intensity are distinguished in the calculation of the section street-road network.

Table 2. The effect of the dew point on the traffic flow characteristics.

\begin{tabular}{lcccccc}
\hline & $\begin{array}{c}\text { Minimum } \\
\text { intensity } \\
(\text { vehicles/h) }\end{array}$ & $\begin{array}{c}\text { Average } \\
\text { intensity } \\
(\text { vehicles/h) }\end{array}$ & $\begin{array}{c}\text { Maximum } \\
\text { intensity } \\
(\text { vehicles } / \mathrm{h})\end{array}$ & $\begin{array}{c}\text { Minimum } \\
\text { average } \\
\text { speed } \\
(\mathrm{km} / \mathrm{h})\end{array}$ & $\begin{array}{c}\text { Average } \\
\text { speed }(\mathrm{km} / \mathrm{h})\end{array}$ & $\begin{array}{c}\text { Maximum } \\
\text { average speed } \\
(\mathrm{km} / \mathrm{h})\end{array}$ \\
\hline Min & 14 & 38 & 1284 & 18.1 & 45.8 & 149.0 \\
Avg & 14 & 28 & 456 & 16.3 & 45.5 & 145.1 \\
Max & 13 & 25 & 300 & 14.2 & 44.1 & 138.6
\end{tabular}

Thus, the effect of the dew point (table 2) is significant: a decrease in the deficit leads to an increase in throughput (average speed and intensity) of traffic flow.

Table 3. The effect of atmospheric pressure on the traffic flow characteristics.

\begin{tabular}{lcccccc}
\hline & $\begin{array}{c}\text { Minimum } \\
\text { intensity } \\
(\text { vehicles/h) }\end{array}$ & $\begin{array}{c}\text { Average } \\
\text { intensity } \\
(\text { vehicles/h) }\end{array}$ & $\begin{array}{c}\text { Maximum } \\
\text { intensity } \\
(\text { vehicles } / \mathrm{h})\end{array}$ & $\begin{array}{c}\text { Minimum } \\
\text { average } \\
\text { speed } \\
(\mathrm{km} / \mathrm{h})\end{array}$ & $\begin{array}{c}\text { Average } \\
\text { speed }(\mathrm{km} / \mathrm{h})\end{array}$ & $\begin{array}{c}\text { Maximum } \\
\text { average speed } \\
(\mathrm{km} / \mathrm{h})\end{array}$ \\
\hline Min & 15 & 23 & 180 & 18.8 & 46.0 & 149.4 \\
Avg & 16 & 36 & 600 & 17.5 & 44.2 & 127.1 \\
Max & 16 & 72 & 1068 & 15.3 & 40.3 & 113.2
\end{tabular}


An increase in atmospheric pressure (table 3) leads to an increase in the traffic flow intensity, but at the same time, the values of average speed decrease, which allows concluding that the traffic flow is in a state of obstructed movement, which increases the density of the streams according to (2).

Table 4. The effect of wind speed on the traffic flow characteristics.

\begin{tabular}{lcccccc}
\hline & $\begin{array}{c}\text { Minimum } \\
\text { intensity } \\
(\text { vehicles/h) }\end{array}$ & $\begin{array}{c}\text { Average } \\
\text { intensity } \\
(\text { vehicles/h) }\end{array}$ & $\begin{array}{c}\text { Maximum } \\
\text { intensity } \\
(\text { vehicles } / \mathrm{h})\end{array}$ & $\begin{array}{c}\text { Minimum } \\
\text { average } \\
\text { speed } \\
(\mathrm{km} / \mathrm{h})\end{array}$ & $\begin{array}{c}\text { Average } \\
\text { speed }(\mathrm{km} / \mathrm{h})\end{array}$ & $\begin{array}{c}\text { Maximum } \\
\text { average speed } \\
(\mathrm{km} / \mathrm{h})\end{array}$ \\
\hline Min & 15 & 41 & 604 & 0 & 46.7 & 150.0 \\
Avg & 15 & 38 & 600 & 0 & 46.6 & 149.2 \\
Max & 1 & 3 & 84 & 0 & 38.4 & 137.7
\end{tabular}

Wind speed (table 4) has a significant effect at maximum values: a decrease in intensity and speed is observed. Experiments were carried out to take into account the joint influence of road conditions on the traffic flow characteristics. For the formation of a dangerous winter slipperiness type of ice, which reduces the average speed and intensity, the following weather conditions are necessary: air temperature from 0 to $-10{ }^{\circ} \mathrm{C}$, an increase in the dew point deficit, and regular changes in wind direction and speed. The greater the wind speed, the more intense the ice. For all sections of the road network, weather conditions were identified under which the likelihood of ice formation increases (table 5).

Table 5. Weather conditions for the occurrence of ice and its consequences.

\begin{tabular}{lcccccc}
\hline & $\begin{array}{c}\text { Dew } \\
\text { point } \\
\left({ }^{\circ} \mathrm{C}\right)\end{array}$ & $\begin{array}{c}\text { Temperatur } \\
\mathrm{e}\left({ }^{\circ} \mathrm{C}\right)\end{array}$ & $\begin{array}{c}\text { Wind } \\
\text { direction }\left({ }^{\circ}\right)\end{array}$ & $\begin{array}{c}\text { Wind speed } \\
(\mathrm{km} / \mathrm{h})\end{array}$ & $\begin{array}{c}\text { Intensity } \\
\text { reduction }(\%)\end{array}$ & $\begin{array}{c}\text { Average speed } \\
\text { reduction }(\%)\end{array}$ \\
\hline Viby Ringvej & 3 & 0 & 180 & 11.5 & 23 & 16 \\
Christian X's Vej & 4 & 1 & 140 & 15.7 & 27 & 18 \\
Marselis & 4 & -2 & 140 & 12.8 & 30 & 22 \\
Boulevard & 5 & -1 & 180 & 10.1 & 22 & 16
\end{tabular}

\section{Conclusion and discussion}

Thus, I propose a method for profiling the impact of road, organizational and weather conditions on traffic flow using a data-driven approach. The method operates with macroscopic characteristics of traffic flow and detailed data on road conditions, represented by the models of objects, processes, and phenomena of the real world. The proposed method is implemented as a software module for the intelligent transport geographic information system. I give the results of the analysis of data on the speed and intensity of traffic flow depending on environmental conditions.

The environment has the greatest adverse effect in winter, characterized by a decrease in the length of daylight, low air temperatures, and road surface. Mutual combination of strong side wind and slippery coating leads to loss of stability in open areas of the street-road network. Winter slippery conditions, snow drifts reduce the speed, reduce the width of the carriageway due to the formation of snow deposits, reduce the throughput capacity of the street-road network. Precipitation, fogs, blizzards lead to the limitation of the visibility distance, which also entails a decrease in speed and a decrease in throughput.

The results of the analysis showed that the method is effective for profiling and predicting the changes taking place in the traffic flow, with a change in the affecting road conditions.

\section{References}

[1] Buch N, Velastin S A and Orwell J 2011 A review of computer vision techniques for the analysis of urban traffic IEEE T-ITS 12(3) 920-939 
[2] Qian Y, Zeng J, Wang N, Zhang J and Wang B 2017 A traffic flow model considering influence of car-following and its echo characteristics Nonlinear Dynamics 89(2) 1099-1109

[3] Lighthill M J and Whitham G B 1955 On kinematic waves: II. Theory of traffic flow on long crowded roads Proc. R. Soc. London Ser. A 229 281-345

[4] Drew D R 1968 Traffic Flow Theory and Control (New York: McGraw Hill Text) p 467

[5] Lukanin V N, Buslaev A P, Trofimenko Yu V and Yashina M V 1998 Road traffic and environment (Moscow: INFRA-M) p 408

[6] Kurzhanskij A B, Kurzhanskij A A and Varajya P 2010 The role of macromodelling in the active management of the transport network MIPT proceedings 2(4) 100-118

[7] Burghout W, Koutsopoulos H N and Andreasson I 2005 Hybrid mesoscopic-microscopic traffic simulation TRR: Journal of the Transportation Research Board 1934(1) 218-255

[8] Hoogendoorn S P and Bovy P H L 2001 State-of-the-art of vehicular traffic flow modelling Proc. of the Institution of Mechanical Engineers I: Journal of Systems and Control Engineering 215(4) 283-303

[9] Cheng R, Ge H and Wang J 2017 An extended macro traffic flow model accounting for multiple optimal velocity functions with different probabilities Physics Letters A 381(32) 2608-2620

[10] Newell G F 1993 A simplifed theory of kinematic waves in highway traffic II: Queueing at freeway bottlenecks Transp. Res. B 27 289-303

[11] Wang F Y 2010 Parallel control and management for intelligent transportation systems: Concepts, architectures, and applications IEEE Transactions on Intelligent Transportation Systems 11(3) 630-638

[12] Kolesov V I, Gulyaev M L and Osipenko A M 2011 Identification of the real model of traffic flow in the city Vestnik of the Orenburg State University 10(129) 43-48

[13] Agafonov A A and Myasnikov V V 2014 An algorithm for traffic flow parameters estimation and prediction using composition of machine learning methods and time series models Computer Optics 38(3) 539-549

[14] Tang T Q, Li P and Yang X B 2013 An extended macro model for traffic flow with consideration of multi static bottlenecks Physica A: Statistical Mechanics and its Applications 392 3537-3545

[15] Zhang J, Wang F Y, Wang K, Lin W H, Xu X and Chen C 2011 Data-driven intelligent transportation systems: A survey IEEE Transactions on Intelligent Transportation Systems 12(4) 1624-1639

[16] Johansson M and Gunnar A 2006 Data-driven traffic engineering: techniques, experiences and challenges 3rd Int. Conf. on Broadband Communications, Networks and Systems, IEEE 1-10

[17] Daganzo C F 1994 The cell transmission model: A dynamic representation of highway traffic consistent with the hydrodynamic theory Transportation Research Part B: Methodological 28(4) 269-287

[18] Golovnin O K and Mikheeva T I 2018 Attribute-driven network-centric urban transport process control system modeling Journal of Physics: Conference Series 1096(1) 012199

[19] URL: http://iot.ee.surrey.ac.uk:8080/datasets.html

[20] Ali M I, Gao F and Mileo A 2015 CityBench: A configurable benchmark to evaluate RSP engines using smart city datasets Int. Semantic Web Conf. 374-389

[21] Ostroglazov N, Golovnin O and Mikheeva T 2018 System analysis and processing of transport infrastructure information CEUR Workshop Proc. 2298144071

[22] Golovnin O, Stolbova A and Ostroglazov N 2019 An analysis of road traffic flow characteristics using wavelet transform Studies in Systems, Decision and Control 199 433-445 